\title{
ON METHODS OF VISUAL FEEDBACK ORGANIZATION IN UNMANNED AVIATION
}

\author{
A. Ksenzov, post-graduate student of the Computer Chair of the Belarusian State University of \\ Informatics and Radio-electronics, M.Sc.
}

\begin{abstract}
This article is devoted to the analysis of existing modes of visual feedback in Unmanned Aviation, indicating specificity and deficiencies. A new approach to the organization of such feedback with the server-side software algorithms is being proposed.
\end{abstract}

\section{INTRODUCTION}

The specific feature of the present time is an extremely fast and intensive development of the robotic systems of military purpose. Ten years was enough for the ideas about the place, the role and the tasks solved by unmanned aviation technical equipment in operations to change completely [1]. Problems solved with the setting up of systems for military use, are quite similar to the problems encountered in the application of unmanned aircraft technology in civilian areas (pipelines monitoring, deliveries, etc.).

\section{PROBLEM STATEMENT}

Let us consider the following situation: let there is a certain flying device (FD), capable to move at a speed from $x$ up to $y$ $\mathrm{m} / \mathrm{sec}$. Let the given FD according to the industrial necessity should have an opportunity to move in some territorial rectangular with the legs $\boldsymbol{a} \mathrm{m}$. long and $\boldsymbol{b} \mathrm{m}$. wide, in a range of heights from $\boldsymbol{h} \mathbf{1} \mathrm{m}$. up to $\mathbf{h} \mathbf{2} \mathrm{m}$. (territorial corridor).

Remote piloting, takes place at controlling of model aircrafts [2] when the operator-pilot, visually observing FD, influences directly on a command radio-line the bodies of change of position of the flying device (ailerons, elevators and rudders, throttle lever). Such operating is possible only at a visual feedback through supervision of a current position of the flying device. This article is devoted to ways of the organization of visual feedback of FD with the operator.

\section{EXISTING WAYS OF THE ORGANIZATION OF A VISUAL FEEDBACK}

In the history of unmanned aviation next ways of the organization of a visual feedback are pointed out:

1. The systems that allow to carry out tracking of the FD travel on TV image transmitted from a board remotelypiloted FD (RPFD). Earlier (the end of the 80s of the last century) it was informed that American mini RPFD "Pointer" was operated in this way [3].
2. The systems that allow to carry out tracking of the FD travel in real time on the screen of the monitor with loadable electronic thematic itinerary of the region with accuracy of onboard system of global positioning GPS [4]

In brief we shall stop at each of these ways.

The first way assumes the presence of high-speed connection between FD and a control server the maintenance of which within the frameworks of the existing technologies imposes essential restrictions on the use of FD in many regions (it is necessary to note that as far as it is known to me experience of such operating in the CIS countries is negative [5]), the advantage of the given method of control is reception by the operator of this FD of a real picture from a location of FD.

The advantage of the second way is the use of existing technologies, however the operator of the FD cannot see a detailed picture from the location of the FD and as a result cannot completely adequately operate the FD, besides, the operator does not see the actual state of affairs on the place, which certainly complicates the operation of the FD.

\section{THE SUGGESTED WAY OF THE ORGANIZATION OF A VISUAL FEEDBACK}

The author of the given article suggests forming a picture on the basis of transmitted by the FD to a server of its current coordinates. The picture to the operator of the FD is formed on the basis of the data saved on a server. The advantage of this approach is in the absence of necessity of maintenance of a high-speed connection channel; there will be an opportunity of generation of more precise picture. The lack of the system is that the operator of the FD does not see the real state of affairs on a place and cannot react to any unexpected disturber. (Can be solved by the FD transfer of the additional signals about disturbers. Author's comment).

The given approach is based on the pictures stored in the Database (DB) that are a view of the district from the FD from various points. Considering the necessity of displaying at least 24 pictures in a second and also the minimal speed of a FD it is easy to receive that in a DB should be stored not less than 
$\frac{a b(h 2-h 1)}{\left(\frac{x}{24}\right)^{3}}$ pictures, in case $\mathrm{x}$ is small enough it is reasonable to speak about

$$
\frac{a b(h 2-h 1)}{s^{3}}
$$

pictures, where $\mathrm{s}$ is a minimal step in space

(frequently there is no necessity to divide pictures from points that are less than $s$ meters far away). We shall explain the received figures: moving even at the minimal possible speed the operator should have an opportunity "to see" the change of location in compliance with his movement for what he should see 24 shots per second of district, that is in a DB there should be at least 24 pictures from a track passed by the FD for 1 second for any territorial points along which he should move. In case the speed of a FD is small enough it is meaningful to display to the user identical pictures. For this purpose it is suggested to split the parallelepiped of the territory by the grid with step equal to a maximum from values $\mathrm{x} / 24$, s. By transfer of coordinates of the FD to the operator the picture of as much as possible close to a FD is offered.

In particular let the grid of splitting has a step of 10 meters. In this case in a DB there should be stored not less than $\underline{a b(h 2-h 1)}$

$10^{3}$ pictures, except for that the speed of work of the FD's operating should allow to receive not less than 24 pictures in a second. In case the product ab (h2-h1) exceeds 10000000000 (quite small territorial corridor with length $100 \mathrm{~km}$, width $1 \mathrm{~km}$, range of heights $100 \mathrm{~m}$ ) the necessary number of records in a DB will exceed 10 million and accordingly time of the response of a DB for inquiry of the user will exceed the necessary for generation on-line of the pictures for FD operating.

\section{ALGORITHMS OF SOFTWARE FUNCTIONING OF THE VISUAL FEEDBACK ORGANIZATION}

Let us examine the following way of the work organization of software: having received a package of the information from a FD (its coordinates and other information) software should calculate to what point of a corridor grid a FD will be closest during the moment of display of the information [ $\ll$ a point of display »] (that is the time spent on data transmission and the time for picture generation should be considered). Then there comes an inquiry to a DB for delivery of a corresponding picture if it is necessary it is corrected then the picture is displayed to the user (fig. 1). The given cycle should be executed not slower than $0.04 \mathrm{sec}$.

Considering such high requirements to the speed of software functioning it is possible to offer optimization of the given algorithm (especially for multiprocessor servers): having received a package from a FD together with the main thread a prediction thread can be started the primary goal of which will be calculation of the prediction of projection points (possible points of display for the following package), and reception of the pictures for these points. In that case at reception by a server of a following package probably the corresponding picture will already be is in cache, which will allow avoiding one of the longest steps of the algorithm connected with operation of reception of a picture from a DB. At the same time the prediction thread can work ahead on some iteration, which will lead to formation of "a tree of pictures" in cache.

In this case the algorithm will look like that one presented on fig. 2 .

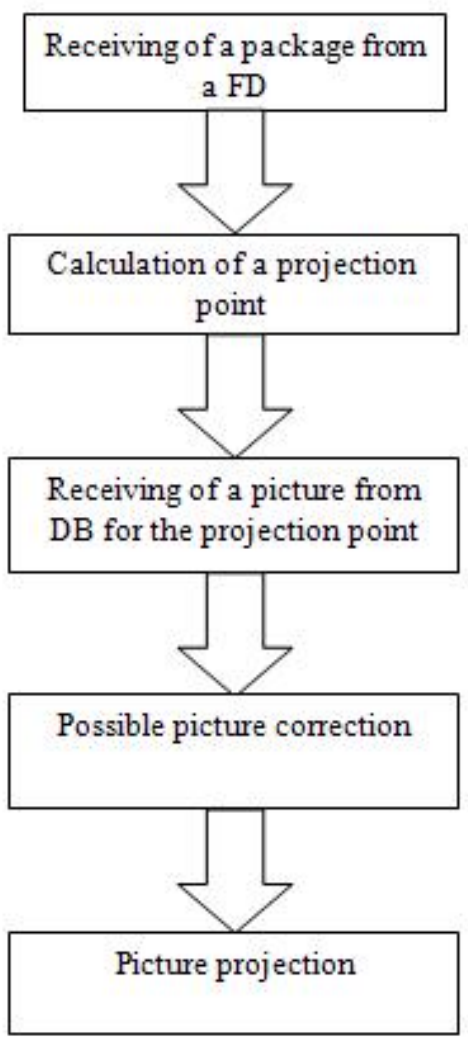

Fig. 1 Algorithm of software work on organization of visual feedback.

\section{THE CONCLUSION.}

In the given article the way of the organization of the visual feedback between the FD and a server of operation has been demonstrated. This way is based on the opportunity to store pictures from various points of spatial corridor splitting on a server in the form of a DB, the opportunity of filling of such $\mathrm{DB}$ is confirmed indirectly by various sources, it is possible to recollect publications in a number of mass-media that concerned the USA construction of virtual model of Baghdad before the beginning of antiterrorist operation on territory of Iraq. In case of existence of the filled DB it becomes possible under the condition of possession of the advanced computer complexes (a set of the corresponding hardware and software) to organize a visual feedback between the FD and its operator on the described algorithms, which enables high-precision multi-purpose FD operating. 


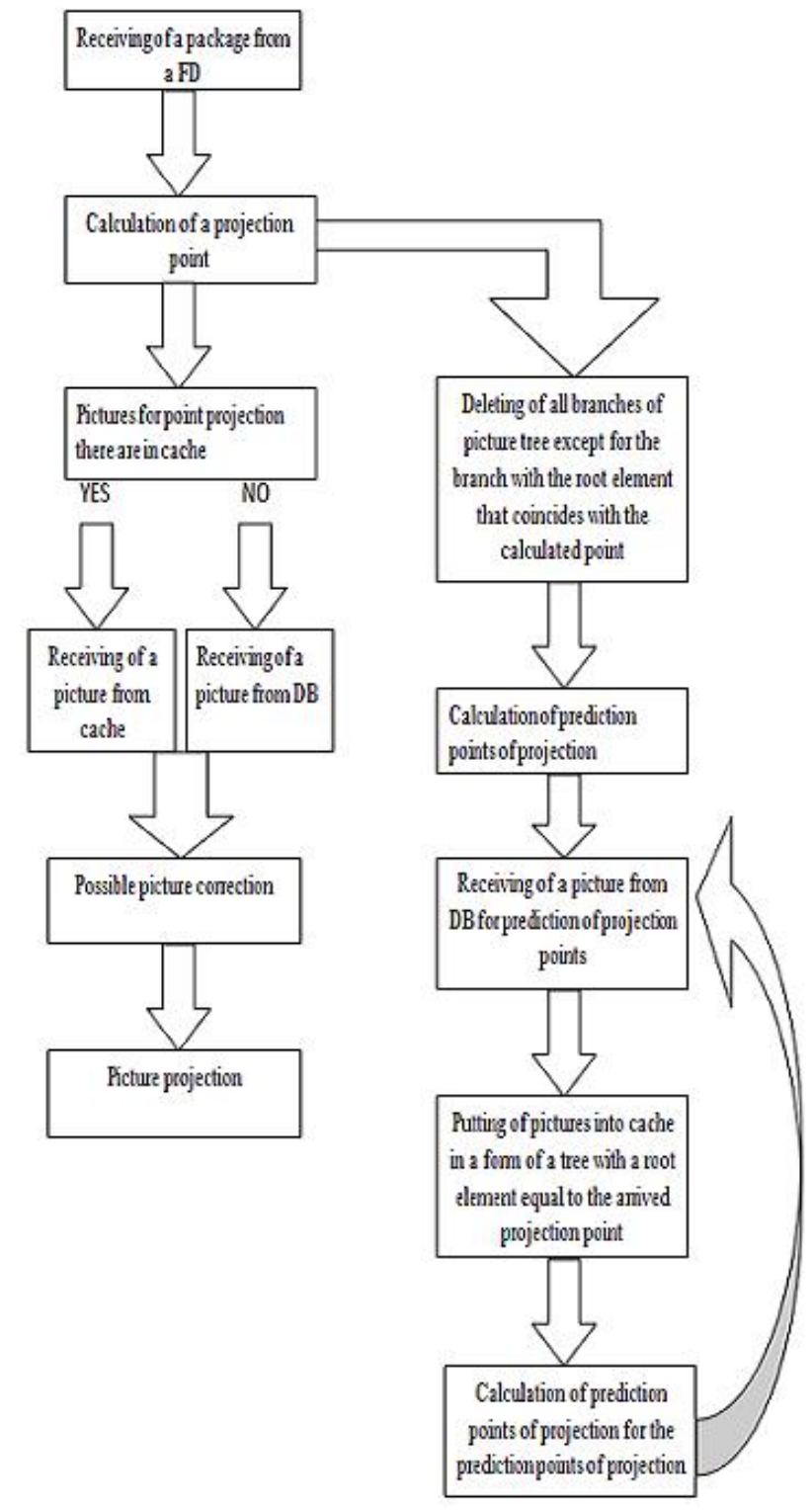

Fig.2 Optimized algorithm of software work on organization of visual feedback.

\section{REFERENCES}

[1] V. Rostopchin. "Unmanned aerial systems. Part 7. Modern classification of unmanned aircraft systems for military use". Available: http://www.avia.ru/author/27.shtml

[2] A. Perfiliev. " The technology of remote controlled model airplanes piloting”. Available: http://www.alfa2.ru:8105/servises/avia/radio/sovet/

[3] "Pointer. UAV Sysytem. AeroViroment Inc" Available: http://www.dpla.ru/Review/Pointer.pdf

[4] T. Kondranin, V. Kozoderov, AG Topchiev, V. Golovko, V. Kosolapov. "Information provision tasks assessment of the state of natural and technogenic scope to the use of space and local monitoring". Available: http://www.iki.rssi.ru/earth/articles06/vol1-185-191.pdf

[5] "Toy photo intelligence XB-39 Eagleye. An attempt of engineering and economic reconstruction". Available :http://dpla.ru/eagleye2.htm 